\title{
RÉFLEXION SUR L'ETHNOMATHÉMATIQUE COMME POSSIBILITÉ PÉDAGOGIQUE
}

\section{ARTICLE ORIGINAL}

SCHWANTES, Vilson ${ }^{1}$

XAVIER, Márcio Pizzete ${ }^{2}$

SCHWANTES, Eloísa Bernardete Finkler ${ }^{3}$

SCHWANTES, Daniel ${ }^{4}$

${ }^{1}$ Master en sciences, mathématiques, UNIJUI - RS. Diplôme et spécialisation en sciences et en mathématiques. Prof. Assistant du CCA - Centre des sciences agronomiques, Campus du maréchal Cândido Rondon, UNIOESTE, PR - Brésil.

${ }^{2}$ Master en développement rural durable-UNIOESTE, Spécialiste en mathématiques, Physique-UNIPAR, Gestion du personnel et éducation spéciale avec une spécialisation en handicap multiple-UNIASSELVI, Diplômé en mathématiques avec une spécialisation en physique-UNIPAR.

${ }^{3}$ Spécialisation en enseignement scientifique exact - Mathématiques, physique et chimie. UNIOESTE - Université d'État de Western Paraná. Graduation: Science et Mathématiques. UNIJUI, RS. Professeur à l'école publique de Paraná.

${ }^{4}$ Professeur de protection des végétaux et de santé humaine au département des sciences végétales de l'Université pontificale catholique du Chili, il occupe un poste interdisciplinaire partagé entre la Faculté d'agronomie et l'Ingeniería Forestal (FAIF), les facultés de médecine et de chimie. Professeur de recherche associé au groupe d'étude sols et environnement (GESOMA - UNIOESTE). Master en agronomie de UNIOESTE, doctorat en agronomie de UNIOESTE (2013-2016) - Période Sandwich (Bourse CAPES) de I'Université de Lisbonne, à I'Instituto Superior de Agronomia (ULisboa). 
JUNIOR, Affonso Celso Gonçalves ${ }^{5}$

KRACKE, Elisa ${ }^{6}$

JUNIOR, Élio Conradi ${ }^{7}$

SCHWANTES, Vilson. Et al. Réflexion sur l'ethnomathématique comme possibilité pédagogique. Revue scientifique pluridisciplinaire de la base de connaissances. 04 année, Ed. 07, vol. 11, p. 148-165. juillet 2019. ISSN: 2448-0959

\section{RÉSUMÉ}

Cet article se penche sur l'ethnomathématique comme une tendance de l'enseignement qui tient compte de la nature culturelle de l'apprentissage mathématique, le contexte social où cette connaissance se développe, le rôle de l'enseignant dans le contexte plus large des relations humaines en supposant Chercheur et médiateur conscient de la pratique pédagogique. La recherche tient compte de l'importance de l'histoire des connaissances mathématiques, de ses

5 Niveau de productivité $1 \mathrm{C}$ du CNPq dans le domaine des sciences de l'environnement avec trois post-doctorants, UEM-PR (Brésil), Université de Santiago de Compostela (Espagne), UFG-GO (Brésil). II est actuellement professeur associé à UNIOESTE-PR et professeur et chercheur au Centre des sciences agraires, où il enseigne la chimie. Chargée de cours au programme de master en sciences agricoles de l'UEM. II est actuellement consultant ad hoc auprès du CNPq, de CAPES et de la Fundação Araucária. Agit à titre de consultant bénévole en environnement auprès de MP-SP et de CONAMA-DF.

${ }^{6}$ Premier cycle en agronomie - UNIOESTE - Université d'État de Western Paraná Diplômé en administration - Unip - Universidade Paulista.

7 Étudiante à la maîtrise en agronomie (production végétale) de l'Université d'État de Western Paraná (UNIOESTE). Ingénieur agronome diplômé d'UNIOESTE (20142018), agit en tant que chercheur associé au groupe d'étude des sols et de l'environnement (GESOMA - UNIOESTE). 
multiples relations avec d'autres domaines d'expertise et de son interdépendance avec les activités quotidiennes. Le texte réfléchit également sur les possibilités et les défis de l'école pour promouvoir des actions pédagogiques qui tiennent compte des relations interpersonnelles dans le processus de (re) construction du savoir. II fait appel à un travail pédagogique critique d'investigation, qui cherche de manière articulée à établir des liens entre les pratiques quotidiennes et les mathématiques scolaires.

Mots-clés : ethnomathématiques, connaissances sociales, compétences mathématiques, activités quotidiennes, pratique pédagogique.

\section{INTRODUCTION}

Au quotidien, les éducateurs continuent de réfléchir aux méthodologies d'enseignement et aux modes d'apprentissage. L'un des principaux défis de l'école continue de contribuer à la formation de citoyens critiques, conscients et actifs dans la société. Une tâche complexe qui exige que les professionnels de l'école et de l'éducation aillent au-delà des programmes d'études, du contenu et du programme d'éducation.

Le monde dans lequel nous vivons, bien que nous ne le réalisions pas, a toujours dépendu fondamentalement des mathématiques. Elle a toujours été et est présente dans pratiquement tout ce qui nous entoure. Son applicabilité est pertinente même dans d'autres sciences, comme l'affirme D'ambràsio (1996, p. 31). "La tendance de toutes les sciences est de plus en plus matematizarem en raison du développement de modèles mathématiques qui développent des phénomènes naturels de manière appropriée."

Comme nous le voyons, les mathématiques sont imprégnées dans tout l'environnement social de l'être humain et sont mises en évidence par chaque nouvel apprentissage. Toutes les professions actuelles s'approprieront les connaissances mathématiques pour créer, maintenir et maintenir des règles, des formules et des conduites. Le maçon, la couturière, le cuisinier, l'ingénieur, les techniciens de toutes 
les professions et les professionnels de tous les secteurs de l'activité humaine dominent certaines connaissances mathématiques, mais n'ont pas toujours appris les calculs pour résoudre leurs problèmes dans les banques école.

Pour Rosa Neto (2002), il existe une relation significative entre les mathématiques et la culture, à la fois en raison de notre adaptation en fonction de nos besoins de survie à travers le temps, représentant à la fois, une empirique, systématique et scientifique.

Travailler l'ethnomathématiques dans l'espace scolaire, selon D'ambrôsio (2008), c'est contribuer aux nouvelles générations à connaître et à reconnaître une mathématique beaucoup plus culturelle, liée à la vie quotidienne de plusieurs groupes ethniques. Pour D'ambrôsio et Rosa (2016), il s'agit d'une posture didactique qui vise à améliorer le processus d'enseignement-apprentissage de la discipline avec l'incorporation dans le programme mathématique des connaissances issues de la vie de l'étudiant et des valeurs humaines, telles que, par exemple, le coopération, la solidarité et l'éthique. Ce sont des connaissances qui favorisent l'appréciation et le respect des différentes façons dont l'humanité utilise quotidiennement pour expliquer, comprendre, comprendre et traiter les situations problématiques rencontrées dans la vie quotidienne.

Avec cette prise de conscience de l'importance et du rôle des mathématiques dans le développement des citoyens et de la société, cet article, en plus de réfléchir, cherche à diffuser les ethnomathématiques, croyant qu'il en tant que proposition pédagogique peut grandement contribuer à Atténuer les difficultés d'apprentissage et le manque d'intérêt de nombreux étudiants pour l'étude de la discipline.

Dans notre compréhension, un travail éducatif centré sur cette perspective, rend l'étudiant se sentent plus en sécurité de sa propre capacité à construire des connaissances mathématiques, dans la recherche de solutions aux problèmes qu'il rencontre dans sa vie quotidienne. Toutefois, pour D'ambrôsio (1996), il est nécessaire que l'enseignant adopte une nouvelle posture en classe, qu'il cherche un nouveau 
paradigme qui remplace l'enseignement-apprentissage déjà usé basé sur une relation obsolète de cause à effet.

Pompée et Monteiro (2001, p. 14), avertissent,

Le changement de paradigme, cependant, est un processus complexe; Il faut vouloir changer et croire que c'est possible. Plus que de constater que nous devons changer, il faut avoir la conviction qu'il y a toujours une nouvelle façon d'enseigner, qu'il est toujours possible de changer.

Les auteurs soulignent que le travail pédagogique dans cette perspective exige que l'enseignant comprenne et clarifie la portée de son rôle, ainsi que l'importance du contenu mathématique avec lequel ils travaillent avec les élèves. En théorie, il est nécessaire de comprendre l'élève d'une manière intégrale, en cherchant à identifier ses besoins de développement au niveau intellectuel, physique, émotionnel, social et culturel. Par conséquent, ils ont souligné que,

Les enseignants actuels sont confrontés à un grand défi : éduqués dans un processus fragmenté, ils devront dépasser les limites que cette formation leur impose et extrapoler les limites du contenu vu de manière partielle et historique (POMPEU et MONTEIRO, 2001, p. 15).

La tâche de l'enseignement et de l'apprentissage, dans cette approche, est en fait différente de celle traditionnellement connue. Cela fait partie de ce que nous croyons être consensuel, c'est-à-dire que toutes les cultures et tous les peuples ont développé leurs propres façons d'expliquer et de modifier leur propre réalité. Ces connaissances et la mise à disposition dans tous les processus de développement humain, propres à chaque culture, sont bien sûr dynamiques, dans la phase d'évolution et de croissance constante, contribuant ainsi à l'élaboration de connaissances systeted.

D'ambrôsio (1993) souligne que les gens ont une culture, [etno]traitent et expliquent leur propre cul[matema]ture, cependant, chacun à sa manière et à sa forme[tica]. Cette constatation a donné lieu au nom ethnomathematics, qui à son tour prend en compte 
les explications des communautés, les égoutteant avec les formes universelles de la connaissance. C'est un fait que chaque enfant avant d'arriver à l'école sait comment faire face parfaitement à sa réalité, qui est en constante évolution.

Selon l'auteur, l'ethnomathématique fait partie du principe et de l'idée d'intégrer les modèles liés à la tradition et à la culture des enfants, en les reconnaissant comme valides pour remplir leurs objectifs. Ainsi, l'ethnomathématique s'en prend à la curiosité de l'enfant, se tourne d'abord vers l'environnement où elle vit, accomplissant une tâche que l'école a oubliée : libérer et développer la créativité des élèves à travers des projets Enquête.

Cette perspective pédagogique est ce qu'on appelle l'ethnomathématique, une ${ }^{[}{ }^{[8]}$ endance de l'enseignement des mathématiques qui considère dans sa pratique pédagogique les aspects sociaux et culturels de l'humanité. II est caractérisé comme une tendance d'enseignement qui apporte à la discussion et cherche à réfléchir sur les différentes formes de Matematizar présents dans notre réalité socioculturelle.

Il propose une éducation critique, dont la base est soutenue dans les études et les écrits d'Ubiratan D'ambrsio, qui[i] pointent vers une proposition méthodologique de l'enseignement mathématique qui valorise les expériences des étudiants. Une vision aux traits qui imprègnent les processus : anthropologique, social et politique, apportant à la discussion la culture locale de chaque groupe social, comme une activité humaine culturellement déterminée par le contexte où elle est réalisée.

C'est dans cet aspect que l'ethnomathématique, au cours des dernières décennies, a été consacrée à l'échelle nationale et internationale comme une proposition didactique-pédagogique pour l'enseignement de la discipline mathématique. Selon D'ambrôsio (2001) «L'ethnomathématique est les mathématiques pratiquées par les groupes culturels, tels que les communautés urbaines et rurales, les groupes de travailleurs, les classes professionnelles, les enfants d'un certain groupe d'âge, les sociétés autochtones et tant d'autres Groupes qui sont identifiés par les objectifs et les traditions communes aux groupes » (p. 9). 
C'est, selon l'auteur, une tendance à l'enseignement où la connaissance se manifeste de manière holistique, c'est-à-dire qu'elle permet aux différents groupes culturels de connaître, de comprendre et d'expliquer leur monde, en utilisant les mathématiques, en développant Capacité de gérer et d'interférer, à travers elle, dans sa réalité. II invite à la possibilité de construire un programme scolaire qui intègre la vie socioculturelle des élèves dans les pratiques pédagogiques, comme l'une des formes d'inclusion sociale.

Comme l'a dit D'ambrôsio (1993), " l'ethnomathématique est un programme qui vise à expliquer les processus de génération, d'organisation et de transmission des connaissances dans divers systèmes culturels et les forces interactives qui agissent dans et entre les trois processus » (p. 7).

Dans cette tendance pédagogique, le programme est dynamique, contextualisé, reconnaît la pluralité culturelle et sociale des étudiants, permet à l'étudiant de devenir une matière active, participative dans le processus de transformation de son environnement local, régional et mondial.

En ce qui concerne le programme d'ethnomathématiques, D'ambrôsio (2001, p. 17) s'interroge également sur le fait que,

La grande motivation du programme de recherche que j'appelle Ethnomathematics est de chercher à comprendre le savoir-faire mathématique tout au long de l'histoire de l'humanité, contextualisé dans différents groupes d'intérêt, communautés, peuples et nations.

Pompée et Monteiro (2001), en ce qui concerne l'adoption d'une pratique pédagogique qui amène le savoir/faire en classe,

Elle est imprégnée de certitudes qui ne sont pas « données » par la science, mais plutôt par les valeurs, les traditions et le respect de ceux qui enseignent. Ainsi, il a une "certitude émotionnelle" confirmée par l'expérience, différente de la "certitude scientifique" qui, en général, est 
sensible à la démonstration rationnelle et les mathématiques, bien que, lorsqu'il est enseigné, aussi instituant des valeurs émotionnelles, présents dans la personne de Enseignant (p. 134 à 135).

Dans l'action pédagogique, la relation à établir entre les processus d'enseignement et d'apprentissage est dialogique, car c'est le contexte de ces interactions qui favorise les possibilités d'apprentissage. Dans cette dynamique, apporter l'ethnomathématique et savoir/faire en classe, c'est récupérer les expériences et les connaissances présentes dans les activités quotidiennes des différentes classes ouvrières, dans un effort pour surmonter le sciencenisme de la connaissance scolaire.

Toutefois, Pompée et Monteiro (2001), en ce qui concerne le travail en classe, les connaissances formelles et informelles ont souligné que,

[...] II est nécessaire pour un groupe de connaître et de reconnaître les limites de ses connaissances pour aller au-delà et chercher le nouveau avec conscience, en connaissant et en discutant de l'importance et de la validité de cette nouvelle. Ce n'est qu'ainsi qu'il saura choisir quand et quelles connaissances utiliser (p. 53-54).

Dans les écoles en général, il est enseigné et appris que les mathématiques dicte comme officiel, tandis que dans les activités quot[comercias, agrícolas, de prestação de serviços, entre outras]idiennes, ils appliquent et utilisent des connaissances mathématiques non officiellement reconnues, mais construit socialement, validé et Apprécié par la société.

En bref, il semble parfois que les citoyens vivent avec l'existence de deux mathématiques: l'école, véc[oficial]ue dans les écoles, théorique, abstraite et sélective et celle de la vie réelle qui répond aux besoins émergents des citoyens. II est perçu que cette " autre mathématique », rarement acceptée par l'école, se montre pratique, dynamique et à l'écoute de tous les secteurs de la société, étant largement utilisé dans les activités courantes des citoyens. 
Selon D'ambrôsio (2001), ethnomathematics, en plus de ne pas nier les mathématiques formelles, cherche à mettre en discussion chaque forme de Matematizar, en analysant argumentativement et réflexivement chaque contexte où les mathématiques sont insérées. II comprend et discute les différentes formes de Matematizar, à condition que les objectifs de chaque contexte soient atteints, de chaque groupe d'égal à égal dans leurs situations spécifiques.

Par conséquent, le travail des mathématiques dans une appr ${ }^{[10]}$ oche ethno, comme le propose D'ambrôsio (1993), exige de l'éducateur d'apprendre à traiter les différentes connaissances culturellement construites et de circuler parmi les élèves. II propose que l'école repense sa pratique, en harmonie avec les aspirations et les besoins quotidiens des gens, en sauvant la fonction sociale de la connaissance mathématique.

Dans cette dynamique, l'éducateur doit être ouvert aux changements dans la façon d'agir et de travailler. Vous devez écouter les expériences que les élèves apportent de la maison, de leur communauté, des relations établies entre eux, en cherchant à partir de ce processus dialogique-interactif pour travailler le contenu mathématique. Et, en plus d'intégrer de nouvelles méthodologies dans leur pratique, constamment analyser et évaluer leur pratique, l'amélioration des actions pédagogiques précédemment expérimentées. Enfin, il doit construire les connaissances mathématiques à partir des expériences personnelles des personnes impliquées dans le processus éducatif.

Ainsi, selon le professeur Ubiratan D'ambrâsio, la pratique de l'ethnomathématique en classe, suggère de suivre un chemin beaucoup plus large de socialisation et de partage de connaissances différentes.

En pratiquant l'ethnomathématique, l'éducateur atteindra les grands objectifs de l'éducation mathématique, avec des perspectives distinctes pour différents environnements culturels et systèmes de production. Il est justifié d'insérer l'élève dans le processus de production de son groupe communautaire et social et témoigne de la diversité culturelle et historique dans différents contextes (D'ambrôsio, 2008, p, 8). 


\section{JUSTIFICATION}

Dans les écoles, dans un pourcentage considérable, il est perçu que le processus d'enseignement-apprentissage des mathématiques continue d'être immuable, c'est-àdire que l'enseignant continue d'apporter l'information prête, que l'élève les reçoit passivement, de façon transparente, dans ces cas, Un conformisme accentué et un déterminisme historique. Dans la pratique, il existe aussi une méthodologie disjointe entre ce qui s'apprend à l'école et ce que la société exige vraiment de ses citoyens.

Selon Santos (2016):

L'absence de contextualisation dans l'enseignement des mathématiques peut provoquer le découragement de la discipline, ressuscitant les méthodes traditionnelles qui conceptualisent les mathématiques comme une science qui a apporté toutes choses prêtes, comme si c'était une connaissance prête et par terre. (p. 5).

Un enseignement qui conduit l'apprenant à un apprentissage mécanique. En outre, il y a encore ceux qui croient que la connaissance mathématique n'est possible que pour les privilégiés, pas accessibles à tous, ce qui, selon Ubiratan D'ambrûsio est comparable au système de "caste" appliqué à certains pays.

[...] Certains éducateurs en mathématiques voient les mathématiques comme une forme privilégiée de connaissances, accessible uniquement à certains particulièrement doués, et dont l'enseignement doit être structuré, en tenant compte du fait que seuls certains esprits, d'une manière "spéciale", peuvent Assimiler et apprécier les mathématiques dans sa plénitud[...]e. (D’AMBRÓSIO, 1996 p.9).

Afin de minimiser cette situation et d'apporter motivation et joie en classe, nous cherchons à travers cette recherche bibliographique, à la fois une mathématique plus contextualisée pour les élèves, et un chemin méthodologique qui donne de meilleurs résultats. Une alternative pédagogique qui permet plus de signification pour le contenu 
des mathématiques étudiées. Quelque chose de motivant, attrayant et qui sert vraiment l'apprenant d'une manière qui lui permet de résoudre les situations-problème d'une manière contextualisée, déduire et prendre des décisions face à sa réalité, la compréhension que, y compris les mathématiques formelles est vivante, est présent Dans notre quotidien.

Une mathématique enseignée à l'école qui est présente dans la réalité de la vie des élèves, qui cherche l'interaction entre l'école et la communauté, dans la possibilité d'être un outil pour transformer l'environnement social. Une connaissance dont la construction et la compréhension sont non seulement possibles pour les mathématiciens, les scientifiques, les maîtres ou les médecins, mais aussi pour les groupes sociaux qui développent ou utilisent dans leurs activités de travail des compétences pour mesurer, calculer, dessiner, créer ou Jouez, en fonction de vos intérêts et de vos besoins.

Cela permet aux élèves de devenir des sujets singuliers et réflexifs, émancipés, confiants et interactifs dans la communauté grâce aux connaissances acquises. Giardinetto (1999), dans ses écrits, s'interroge sur le fait que le sujet ne deviendra un citoyen participant de sa société qu'en « s'appropriant les instruments culturels » (p. 73).

Nous savons et convenons que les mathématiques sont une science présente à la fois dans la vie quotidienne des citoyens et dans les centres de recherche. Par conséquent, compte tenu de cette portée, nous ne pouvons pas limiter l'enseignement de cette discipline à une simple mémorisation des règles, des techniques et de la connaissance formelle des définitions, parce que "... L'enseignement n'est pas transférer des connaissances, mais créer des possibilités pour sa propre production ou sa construction. (FREIRE, 1996, p. 52).

Enseigner les mathématiques du point de vue de l'ethnomathématique devient impossible sans sauver les connaissances et les valeurs que les élèves apportent de la maison. Pour que cet acte se réalise, il est indispensable que l'enseignant écoute 
ses élèves, comme c'est indispensable à l'élève qui parle à son professeur et à ses collègues.

Selon l'éducateur Paulo Freire,

"La construction de relations dialogiques sous les fondements de l'éthique universelle des êtres humains, en tant que pratique humaine spécifique implique la conscience des êtres humains, afin qu'ils puissent réellement s'insérer dans le processus historique en tant que sujets qui font leur Propre histoire. " (FREIRE, 1996, p10.)

L'éducateur mathématique Paulus Gerdes, dans ses diverses publications adopte toujours un discours qui sauve les traditions mathématiques des groupes culturels, qu'avant la colonisation étaient selon lui "caché" et imposé par les conventions eurocentriques dans société, ainsi que dans l'espace scolaire.

Ainsi, dans cette perspective , (GERDES, 2010, p. 142) rapporte que

L'ethnomathématique est le domaine de recherche qui étudie les relations et les interconnexions à multiples facettes entre les idées mathématiques et d'autres éléments culturels et constituants, tels que la langue, l'art, l'artisanat, la construction et l'éducation. C'est le domaine de recherche qui étudie l'influence des facteurs culturels sur l'enseignement et l'apprentissage des mathématiques.

Selon l'auteur (2010, p. 142-143) « L'ethnomathématique montre que les idées mathématiques existent dans toutes les cultures humaines, dans les expériences de tous les peuples, de tous les groupes sociaux et culturels, hommes et femmes. " Dans cette perspective culturelle, il est également démontré que

[...] Tous les peuples de tous les temps peuvent contribuer à cette mathématique universelle. Tous les peuples ont le droit d'apprendre et de profiter des connaissances accumulées et de contribuer à leur 
enrichissement. C'est là qu'est une dimension éthique et morale de la réflexion ethnomathématique. (GERDES, 2010, p.144)

Plusieurs auteurs ont déjà rapporté et par notre expérience professionnelle, nous avons également remarqué que les différentes formes de Matematizar sont si présents dans la vie quotidienne des citoyens, que beaucoup de gens les utilisent dans leurs tâches quotidiennes, même si elles n'ont jamais fréquenté une école. Cette connaissance, parfois acquise empiriquement, est d'une valeur et d'une richesse extraordinaires si importantes que beaucoup n'ont pas toujours la sensibilité de les percevoir. Nous avons partagé avec Pompée et Monteiro (2001) quand ils postulent que,

« L'homme se rapporte au monde qui l'entoure, il l'a compris comme une vie mondiale, c'est-à-dire le monde dans lequel les gens sont à l'intérieur, ce qui nous induit par des influences qui nous atteignent à travers nos relations avec lui » (p. 21).

Cependant, il n'est pas prévu, dans cette perspective pédagogique, de proposer une nouvelle mathématique, mais de réfléchir sur la possibilité d'identifier, dans les différents groupes culturels, leurs modes de Matematizar, de valoriser et de considérer leurs façons de calculer, Connaître, expliquer et comprendre le monde qui les entoure, enrichissant dans cette dynamique, les mathématiques scolaires. Comme d'ambrosius (2001) rapports, c'est une mathématique qui "... II fait partie du quotidien ", du Savoirfaire (p. 25). En fait, une proposition pédagogique qui fonctionne de manière intégrée les connaissances formelles et informelles.

Nous croyons qu'à partir du moment où nous considérons l'ethnomathématique comme une alternative pédagogique qui considère les mathématiques dans différents environnements sociaux, culturels et historiques, de nouvelles méthodologies sont incorporées dans l'enseignement. II devient l'école de mathématiques un instrument pour expliquer et comprendre le développement et la production des connaissances générées dans ces différents contextes, motivant les élèves. 
Comprendre les connaissances qui sont développées au sein d'une communauté donnée, c'est comprendre les ethnomathématiques dans cet environnement, c'est-àdire regarder en arrière les diverses formes de manifestation de la connaissance, non seulement de la connaissance mathématique, mais La connaissance de la vie, de l'organisation, de la construction et de la structuration de ces connaissances et de faire qu'ils sont la naissance de ces personnes.

Cependant, de nombreux éducateurs en mathématiques, peut-être en raison de leur éducation universitaire centrée sur la ligne Technicist, montrent des difficultés à rompre avec l'ancien paradigme et l'adoption d'une méthodologie d'enseignement qui interconnecte les mathématiques scolaires avec les mathématiques de vie. Ce fait devient un obstacle pour la salle de classe à transformer en un espace de discussion et d'analyse critique des connaissances socio-culturellement construites. Pour Pompée et Monteiro (2001, p. 19), il est nécessaire de chercher des moyens de surmonter les conflits existants, car «nous vivons un moment où la fragmentation des connaissances limite la compréhension de la réalité ».

Nous devons être convaincus que ce que les élèves font et apprennent à l'école peut être plus aligné sur les actions et les besoins des divers secteurs de l'activité humaine en dehors des murs de l'école. L'éducateur incorpore simplement et apporte à la salle de classe ces connaissances socialement construites. Nous partageons cette recherche avec les écrits de D'Ambrosio et Rosa, car ils considèrent l'ethnomathématiques comme un programme de recherche en histoire et philosophie des mathématiques, avec des implications pédagogiques,

Ainsi, ce programme de recherche représente une méthodologie de recherche qui cherche à analyser les pratiques mathématiques locales, car il cherche à valoriser, diffuser et respecter les connaissances mathématiques (idées, notions, procédures, processus et pratiques) qui Originaire de divers contextes culturels tout au long de l'histoire (D'ambrâsio et ROSA, 2016, p. 17). 
Un travail pédagogique centré sur cette perspective, valorise l'être humain et ses connaissances informelles constituées dans et par la culture où il vit, peut être le début d'un changement dans l'enseignement de cette discipline. D'après D'ambràsio (2001), un enseignement qui « révèle les pratiques saisies en dehors de l'environnement $s[$ pode]colaire contribue à une vision critique de la réalité » (p. 23).

L'école en tant qu'institution qui représente la continuation de la famille dans son contexte plus large, doit comprendre que tout le processus d'enseignementapprentissage qui fait partie des expériences antérieures des citoyens, des formes de matematizar vécues par les élèves en dehors de la L'école, enrichit la loi éducative. Comme l'a dit Giardinetto (1999) "La vie quotidienne elle-même a besoin d'interférences du non-quotidien » (p. 7).

En ce sens, il laisserait travailler les mathématiques officielles comme les seules mathématiques qui mérite l'attention. Nous examinerions et apporterions à la discussion les expériences et les connaissances des élèves avant la phase scolaire. L'école en fusionnant dialogiquement toutes ces connaissances transforme la salle de classe en laboratoire d'apprentissage permanent.

Dans cette tendance, l'un des rôles des éducateurs est de stimuler le désir naturel des élèves d'étudier les différentes mathématiques utilisées dans les différents groupes culturels. En comprenant les processus de Matematizar de chaque culture, ils comparent ces connaissances avec les connaissances formelles, systate et validées par l'école, ayant la possibilité d'organiser leur propre pensée, comprenant à travers cette réflexion l'importance culturelle et social de toutes les connaissances mathématiques circulant dans le monde de la vie. Selon d'ambrôsio (2001), on pourrait mieux comprendre que "le comportement de chaque individu, associé à ses connaissances, est modifié par la présence de l'autre » (p. 32).

Selon D'ambrôsio (1993), enseigner les mathématiques dans cette conception signifie respecter ce que l'élève sait déjà, consiste à reconnaître les connaissances historiquement construites dans les cultures, à établir des moments dialogiques de 
questions critiques qui renouvellent et Les connaissances antérieures de tous les participants au processus d'apprentissage.

Pour D'ambrôsio (2001) II respecte et valorise chacune des mathématiques existantes, et "... C'est la communication qui permet de définir des stratégies d'action commune »(p. 58). L'école, par réflexion dialectique, ${ }^{[11]}$ explique les modes de production des significations mathématiques produites en dehors de son contexte spécifique. Dans cette approche, c'est comme si les mathématiques de l'école se joignait aux formes de matematizar du monde environnant, plutôt que de le remplacer. Alors que les mathématiques scolaires ignorent ou ne discutent pas de cette diversité de connaissances, produite dans la culture de chaque peuple, les valeurs ethnomathématiques et apporte au débat ces différences, en reconnaissant leurs modèles mathématiques et les connaissances qui leur sont liées .

Selon D'ambrôsio (2001), alors que les mathématiques traditionnelles et son enseignement cherchent à universaliser les concepts, les rendant de plus en plus abstraits et généralisants, ethnomathématiques cherche à les réorganiser, les rendant plus spécifiques et applicables à Contexte de la réalité vécue. II propose, en résumé, une façon plus cohérente de marcher. II cherche à amener les mathématiques d[da escola]es livres à la vie et à la vie à l'école, en reconnaissant que les deux ont une histoire et une importance pour comprendre la réalité dans toutes ses dimensions.

L'idée de base de cette tendance à enseigner les mathématiques, selon D'ambrôsio (2001), est de considérer les expériences des étudiants, des parents, des groupes d'égal à égal, des cultures différentes et de toutes les formes d'automatematizar de chaque peuple. Ce sont des connaissances produites par la nécessité pratique de résoudre des problèmes spécifiques de justesse, les petits agriculteurs, les maçons, les charpentiers, les groupes autochtones, les colons, etc. Ce sont des connaissances différentes produites par différents groupes à des situations problématiques éloidéales. 
Pour Pompée et Monteiro (2001), une pratique pédagogique qui s'oppose en même temps à la fragmentation des connaissances se caractérise par la localisation culturelle des connaissances historiques et l'opportunisme des hommes à la rescousse de leur capacité dialogique réfléchie critique. II comprend, selon les auteurs, l'être humain dans son intégralité et la connaissance dans toute sa complexité.

Selon Pompée et Monteiro (2001), un enseignement qui place «l'homme et non le capital comme valeur centrale des relations sociales »(P. 26). Selon D'ambrôsio (2001), un travail centré sur cette perspective exige que les éducateurs aient une bonne dose d'humanisme, parce que " l'élève est plus important que les programmes et le contenu » (p. 86).

Mathématiques travaillé sur une approche ethno apporte à la salle de classe des expériences qui nécessitent la communicabilité et le dialogue argumentatif. Cette posture didactique, à son tour, conduit les personnes impliquées dans le processus éducatif à une croissance personnelle et collective, rendue possible par l'action réfléchie permanente des deux, et des deux pratiques.

Ensemble, les enseignants et les élèves enseignent et apprennent simultanément, connaissent le monde dans lequel ils vivent de façon critique et établissent des relations de respect mutuel, de justice, constituant un véritable climat de discipline, par des relations dialogiques, faisant de la salle de classe un défi Intéressant et stimulant pour toutes les personnes impliquées. "Celui qui enseigne apprend quand on enseigne et qui apprend enseigne dans l'apprentissage » (FREIRE, 1996, p. 38).

Selon Giardinetto (1999), " l'enseignant peut et doit utiliser les connaissances quotidiennes comme un point de soutien pour le processus d'enseignementapprentissage » (p. 68), amenant l'élève dans le domaine de l'argumentation, développant l'habitude de la lecture critique, de la recherche, Questionnement, de créativité, indispensable à la formation des citoyens.

En lançant ce regard sur leur pratique, les connaissances antérieures des étudiants sont valorisées et d'autres sont formées, toujours à partir du connu. L'important est 
l'établissement de liens de connaissance de la communauté avec la connaissance de l'école et la connaissance de l'école avec la connaissance de la communauté. Grâce à cet établissement et à ces relations, il est possible d'attribuer un sens aux deux connaissances. Dans ce contexte, dans ce contexte, la connaissance présente un caractère dynamique, toujours ouvert à de nouvelles approches.

Pour Freire (1996) Le rôle de l'enseignant est lié à la conception que l'enseignement n'est pas le transfert des connaissances, mais la création des conditions pour sa construction. Cela signifie reconnaître qu'ensemble, les élèves et les enseignants apprennent en classe, car ils apportent tous de nombreuses connaissances des expériences scolaires et non scolaires qu'ils ont vécues au cours de leur existence.

Nous croyons que l'ethnomathématique peut aider chaque éducateur dans cette possibilité pédagogique de remplacer les actions de routine de «donner des classes prêtes» par une posture qui (re) orienter l'apprentissage de l'élève dans la construction de ses propres connaissances, fruit de Interaction entre les sujets dans ce processus dialogique. Même parce que la société de la connaissance dans laquelle nous vivons, exige aujourd'hui une position holistique dans la formation de ses citoyens, qui sont capables d'interagir avec le dynamisme dans tous les contextes, construire de nouvelles significations pour les mathématiques, explorer Différentes formes de situations-problème de diverses activités humaines.

\section{BUT}

Contribuer de la réflexion à l'éducateur en mathématiques pour adopter une posture critique d'investigation par rapport à l'enseignement des mathématiques, de la perspective d'initier son activité d'enseignement à travers l'établissement de liens entre les mathématiques quotidiennes et Mathématiques scolaires.

\section{MÉTHODOLOGIE}

Les inspirations théoriques de cet article sont dues à des lectures et des réflexions sur l'éducation mathématique, l'ethnomathématique et la perspective interculturelle de 
l'enseignement. Les écrits cherchent à réfléchir sur l'approche ethnomathématique dans la perspective qu'un enseignement basé sur les connaissances antérieures des étudiants, valorisant leur culture et leurs modes de Matematizar, sera un facteur agrégation de la connaissance, donnant un caractère plus humain Pour la discipline.

\section{CONTRIBUTIONS ATTENDUES}

Puisse-t-on percevoir, selon D'ambrôsio (2001), l'importance de l'ethnomathématique dans la formation de l'étudiant, puisque son parti pris culturel et anthropologique indique le chemin qui mène au respect des différences, de la compréhension et de l'inclusion, si nécessaires à notre réalité vie sociale.

Que l'enseignement des mathématiques en classe, dans cette perspective pédagogique, permet un dialogue efficace entre les connaissances mathématiques produites dans différents contextes et que les enseignants réfléchissent davantage sur la diversité culturelle de la discipline et de ses Importance pour l'éducation Systate.

Que, à partir de cette réflexion, la pratique pédagogique en classe puisse être réorientée vers le développement de la pleine citoyenneté des élèves. Que, selon Pinheiro et Rosa (2016), le:

[...] Les professeurs de mathématiques s'immergent dans la dynamique culturelle des élèves et utilisent des stratégies d'enseignement et d'apprentissage qui valorisent la dimension culturelle en classe, de sorte qu'une éducation mathématique inclusive puisse être développée qui pourrait effectivement Contribuer à la transformation sociale (p. 79).

Que cet effort puisse contribuer à de nouveaux éléments pour enseigner la discipline des mathématiques, permettant en classe d'étudier de plus en plus les mathématiques et la réalité, comme des moyens de connaître et d'interpréter chaque contexte, dans un processus dans lequel l'enseignant sera Constamment (re) produire, (re) construire, (re) signifier la connaissance et la connaissance. 
Nous espérons également que les lecteurs perçoivent la possibilité d'insérer dans l'enseignement des mathématiques un regard différencié sur la réalité et sur les mathématiques elles-mêmes et que ce regard corroder avec l'apprentissage mathématique de l'éducation afin de vous emmener à participer à Discussions sur les mathématiques à l'intérieur et à l'extérieur de la salle de classe.

Nous voulons que la salle de classe, basée sur la méthodologie d'enseignement, commence à intégrer / analyser dans le processus pédagogique, les situations problématiques dont la résolution prend en compte à la fois les mathématiques formelles et les mathématiques informelles. Que la discipline soit perçue comme une activité humaine, motivant encore plus l'intérêt des élèves.

Nous soulignons qu'il ne s'agit pas de remplacer les mathématiques par une autre, mais de respecter les différents modes de Matematizar dans différents contextes, dans les buts auxquels ils sont proposés. Ainsi, nous croyons que cette approche éducative peut nous orienter vers une éducation contextualisée, enrichie, motivant les participants à faire partie du processus.

Selon RAM one,

[...] Enseigner les mathématiques dans cette conception permettra à l'élève de lier les concepts travaillant en classe à leur expérience quotidienne, en fonction de leur environnement naturel, social et culturel. II ne s'agit pas de rejeter les mathématiques académiques, mais plutôt d'intégrer des valeurs vécues dans les expériences de groupe, compte tenu des liens historico-culturels (CARNEIRO, 2012, p. 3).

Enfin, il entend contribuer à la Constitution du chercheur Professeur de sa pratique, l'éveillant à apprendre de manière continue à être enseignant, en fonction des besoins éducatifs actuels, transformant la salle de classe en un environnement d'apprentissage Échange démocratique de connaissances, sauvetage de la connaissance historique-socialement construite de l'humanité. 


\section{RÉFÉRENCES BIBLIOGRAPHIQUES}

CARNEIRO, K. T. A. Cultura Surda na aprendizagem matemática da sala de recurso do Instituto Felipe Smaldone: uma abordagem etnomatemática. Anais do 4ํㅡㄹ Congresso Brasileiro de Etnomatemática. Belém, PA: ICEm4, 2012.

D'AMBRÓSIO, Ubiratan. O programa Etnomatemático: Uma síntese. Acta Scientía, v.10, n.1, Jan/jun.2008.

Etnomatemática - elo entre as tradições e a modernidade. Coleção Tendências em Educação Matemática, 1. Belo Horizonte: Autêntica, 2001, 112p.

- Da realidade a ação: Reflexões sobre a educação e matemática. Campinas: Unicamp, 1996.

. Etnomatemática: Arte ou técnica de explicar e conhecer. Editora Ática, Série Fundamentos, 2. edição, São Paulo, 1993.

D'AMBROSIO, U.; ROSA, M. Um diálogo com Ubiratan D'Ambrosio: uma conversa brasileira sobre etnomatemática. In BANDEIRA, F. A.; GONÇALVES, P. G. F. (Orgs.). Etnomatemáticas pelo Brasil: aspectos teóricos, ticas de matema e práticas escolares. Curitiba, PR: Editora CRV. 2016. pp. 13-37.

FREIRE, Paulo. Pedagogia da autonomia: Saberes necessário a prática educativa. São Paulo: Paz e Terra, 1996.

GERDES, Paulus. Geometria dos Trançados Bora na Amazônia Peruana, Livraria da Física, São Paulo, 2010, 190 p. São Paulo: Editora Livraria da Física, 2011, 190p.

GIARDINETTO, José Roberto Boettger. Matemática escolar e matemática da vida cotidiana. Coleção polêmicas do nosso tempo, autores associados, Campinas - São Paulo, 1999, 128p. 
MONTEIRO, A.; Pombeu, G. Jr. A matemática e os temas transversais. Editora Moderna, São Paulo, 2001, 160p.

PINHEIRO, R. C.; ROSA, M. Uma perspectiva etnomatemática para o processo de ensino e aprendizagem de alunos Surdos. RPEM, v. 5, n. 9, p. 56-83, 2016.

ROSA NETO, R. Didática da matemática, São Paulo: Ática, 2002.

SANTOS, Lijecson Souza dos. Contextualização Matemática Em Situação De Ensino E Aprendizagem No Eja. Disponível em: https://editorarealize.com.br/revistas/cintedi/trabalhos/TRABALHO_EV060_MD1_SA 18_ID 492_23102016194610.pdf. 2016.

8. Dans ce travail d'investigation, utilisé pour désigner l'orientation méthodologique qui peut être développé dans la perspective de l'étude des conceptions, des traditions et des pratiques mathématiques d'un groupe social, à travers lequel le groupe interprète et codifie son connaissance.

9. Ubiratan D'ambràsio, professeur, éducateur en mathématiques et chercheur à I'Université d'État de Paulista (UNESP). Campus Rio Claro. Institut des géosciences et des sciences exactes (IGCE). Il est professeur émérite à l'Université d'État de Campinas/UNICAMP. II a publié des études dans: Histoire et philosophie des mathématiques, I'histoire et la philosophie des sciences, ethnomathématiques, ethnosciences, éducation mathématique et études transdisciplinaires.

10. L'approche ethno se réfère à plusieurs perspectives en circulation concernant ce domaine, qui n'est pas, à ce jour, théoriquement cristallisé. Les éducateurs et les chercheurs impliqués dans cette question ont décidé de donner le temps aux explorations de progresser et de contribuer ainsi à une éventuelle élaboration théorique des ethnomathématiques. II est possible que ce développement intègre largement les différentes perspectives en circulation. 
11. Dialectique dans cette réflexion, exprime l'art de la recherche du dialogue, à travers l'argumentation et contrer l'expansion des idées et des concepts impliqués dans la discussion. Dans l'analyse des faits et des situations-problème qui permettent le développement de la pensée, l'enseignant et l'élève ont une participation dialogique égale, l'un toujours à l'écoute de l'autre, (re) construire ensemble leurs apprentissages.

Soumis : juin 2019.

Approuvé : juillet 2019. 